Research Article

\title{
A CDFT-Based Computational Peptidology (CDFT-CP) Study of the Chemical Reactivity and Bioactivity of the Marine-Derived Alternaramide Cyclopentadepsipeptide
}

\author{
Norma Flores-Holguín $\mathbb{D}^{1},{ }^{1}$ Juan Frau $\mathbb{D}^{2},{ }^{2}$ and Daniel Glossman-Mitnik ${ }^{1}{ }^{1}$ \\ ${ }^{1}$ Laboratorio Virtual NANOCOSMOS, Departamento de Medio Ambiente y Energía, \\ Centro de Investigación en Materiales Avanzados, Chihuahua 31136, Mexico \\ ${ }^{2}$ Departamento de Química, Facultad de Ciencias, Universitad de las Islas Balears, Palma de Mallorca 07122, Spain \\ Correspondence should be addressed to Daniel Glossman-Mitnik; dglossman@gmail.com
}

Received 16 June 2021; Accepted 26 August 2021; Published 6 September 2021

Academic Editor: Wagdy Eldehna

Copyright ( 92021 Norma Flores-Holguín et al. This is an open access article distributed under the Creative Commons Attribution License, which permits unrestricted use, distribution, and reproduction in any medium, provided the original work is properly cited.

\begin{abstract}
Alternaramide is a cyclic pentadepsipeptide isolated from marine sources that has been shown to present weak antibiotic activity against Bacillus subtilis and Staphylococcus aureus as well as inhibitory effects on inflammatory mediator expressions. Thus, this work reports the results of a computational study of the chemical reactivity and bioactivity properties of this cyclopentadepsipeptide considering a CDFT-based computational peptidology (CDFT-CP) methodology that results from the combination of the chemical reactivity descriptors that arise from conceptual density functional theory (CDFT) together with some cheminformatics tools that can be used to estimate the associated physicochemical parameters, to improve the process of virtual screening through a similarity search, and to identify the ability of the peptide to behave as a potential useful drug, complemented with an analysis of its bioactivity and pharmacokinetics indices related to the ADMET (absorption, distribution, metabolism, excretion, and toxicity) features. The results represent a new confirmation of the superiority of the MN12SX density functional in the fulfilment of the Janak and ionization energy theorems through the proposed KID procedure. This has been useful for the accurate prediction of the CDFT reactivity descriptors that help in understanding the chemical reactivity. The computational pharmacokinetics study revealed the potential ability of alternaramide as a therapeutic drug by interacting with GPCR ligands and protease inhibitors. The ADMET indices confirm this assertion through the absence of toxicity and good absorption and distribution properties.
\end{abstract}

\section{Introduction}

There are many studies showing that marine cyclodepsipeptides have a broad spectrum of biological functions, spanning from antitumor, anthelmintic, insecticidal, antibiotic, antifungal, immunosuppressant, anti-inflammatory, anti-HIV to antimalarial activities [1-8].

Alternaramide is a cyclic pentadepsipeptide that has been isolated from an extract of the marine-derived fungus Alternaria spp. SF-5016 whose structure was determined by analysis of the NMR spectroscopic data. It has been shown that alternaramide present weak antibiotic activity against Bacillus subtilis and Staphylococcus aureus [9]. Another study demonstrated that the alternaramide cyclopentadepsipeptide displays inhibitory effects on inflammatory mediator expressions [10].

The first synthesis of alternaramide was presented by Horton and coworkers using solution phase coupling protocols and macrolactonization and macrolactamization routes while its structure was determined by single crystal $\mathrm{X}$-ray analysis [11].

In light of these potential therapeutic properties, we present the results of a computational study of the chemical reactivity and bioactivity properties of this cyclopentadepsipeptide using a CDFT-based computational peptidology (CDFT-CP) methodology [12-20], which is 
based on the combination of chemical reactivity descriptors derived from conceptual density functional theory (CDFT) [21-26], together with some cheminformatics tools [27-34] that can be used to estimate the associated physicochemical parameters, to improve the process of virtual screening through a similarity search, and to identify the ability of the peptide to behave as a potential useful drug, complemented with an analysis of its bioactivity and pharmacokinetics indices related the ADMET (absorption, distribution, metabolism, excretion, and toxicity) features $[35,36]$.

\section{Materials and Methods}

2.1. Density Functional Theory (DFT) Calculations. The Kohn-Sham (KS) methodology entails determining the molecular energy, electronic density, and orbital energies of a given system, particularly the frontier orbitals HOMO (highest occupied molecular orbital) and LUMO (lowest unoccupied molecular orbital), which are intrinsically linked to the chemical reactivity of molecules [37-40]. This methodology is convenient when thinking of quantitative qualities related with conceptual DFT descriptors [21-26]. The definitions for the global reactivity descriptors are as follows [21-26]: electronegativity as $\chi=1 / 2\left(\varepsilon_{L}+\varepsilon_{H}\right)$, global hardness as $\eta=\left(\varepsilon_{L}-\varepsilon_{H}\right)$, and electrophilicity as $\omega=\left(\varepsilon_{L}+\varepsilon_{H}\right)^{2} / 4\left(\varepsilon_{L}-\varepsilon_{H}\right)$. In these definitions, $\varepsilon_{H}$ and $\varepsilon_{L}$ represent the HOMO and LUMO energies related to the marine cyclopentadepsipeptide studied through this research. In turn, the electrodonating power is defined as $\omega^{-}=$ $\left(3 \varepsilon_{H}+\varepsilon_{L}\right)^{2} / 16 \eta$ and the electroaccepting power is defined as $\omega^{+}=\left(\varepsilon_{H}+3 \varepsilon_{L}\right)^{2} / 16 \eta$ while the net electrophilicity is a combination of the previous reactivity descriptors defined as $\Delta \omega^{ \pm}=\omega^{+}+\omega^{-}$.

These global reactivity descriptors arising from conceptual DFT [21-26] are complemented by a new nucleophilicity index $N$ which was established [41-45] considering the value of the HOMO energy obtained by means of the KS scheme using an arbitrary shift of the origin with tetracyanoethylene (TCE) as a reference.

The quality of a density functional can be determined by comparing the results it produces with experimental values or results produced from high-level computations. However, due to lack of experimental results for the chemical systems under investigation or the large size of the molecules, this comparison is not always computationally feasible. A methodology called KID (Koopmans in DFT) has been developed by our research group [12-20] for the validation of a given density functional in terms of its internal coherence. It has been shown that within the generalized Kohn-Sham (GKS) version of DFT, there are some relations between the KID indices and the Koopmans and ionization energy theorems, by connecting $\varepsilon_{H}$ to $-\mathrm{I}, \varepsilon_{L}$ to $-\mathrm{A}$, and a combination of both orbital energies through the formulas $J_{I}=\left|\varepsilon_{H}+E_{g s}(N-1)-E_{g s}(N)\right|, \quad J_{A}=\mid \varepsilon_{L}+E_{g s}(N)-E_{g s}$ $(N+1) \mid$, and $J_{H L}=\sqrt{J_{I}^{2}+J_{A}^{2}}$. An additional KID descriptor $\triangle \mathrm{SL}$ amounting to the difference in energies between the SOMO (equivalent to the HOMO of the radical anion) and the LUMO of the neutral system has been designed to help in the verification of the accuracy of this methodology [12-20].
The conformers of the peptide studied in this study were determined using MarvinView 17.15 from ChemAxon (http://www.chemaxon.com), which was used to perform molecular mechanics calculations using the overall MMFF94 force field [46-50]. Following that, the density functional tight binding (DFTBA) methodology was used to optimize the geometry and calculate the frequency [51]. This final step was required to ensure that there were no imaginary frequencies as a check for the optimized structures' stability as a minimum in the energy landscape. The electronic properties and the chemical reactivity descriptors of the alternaramide marine cyclopentadepsipeptide considered the MN12SX/ Def2TZVP/H2O model chemistry [52-54] on its optimized molecular structure, owing to the fact that has been previously proved that it verifies the "Koopmans in DFT" (KID) procedure [13-20, 55-62], with the aid of the Gaussian 16 software [51] and the SMD solvation model [63]. This model chemistry considers the MN12SX screened-exchange density functional [52] together with the Def2TZVP basis set $[53,54]$ and the charge of the molecule being equal to zero while the radical anion and cation have been considered in the doublet spin state.

\subsection{In Silico Pharmacokinetics Analysis and ADMET Study.} The first step in understanding the potential therapeutic properties of the considered marine cyclopentadepsipeptide was to feed its SMILES (simplified molecular input line entry specification), which was obtained through ChemDoodle 11.3.0 software, into Chemicalize, by ChemAxon (http:// www.chemaxon.com), which was used for the prediction of several properties related to cheminformatics (http:// chemicalize.com/) (accessed March 2021).

The online Molinspiration software from Molinspiration Cheminformatics (https://www.molinspiration. com/) (accessed March 2021) was used to search the chemical universe for molecules having molecular structures that are comparable to the one being analyzed for similarity with already known biological and pharmacological properties for the prediction of the bioactivity scores for the different drug targets.

SwissTargetPrediction is a useful tool that is available online for the efficient prediction of protein targets of small molecules and has been considered for the determination of the potential bioactivity of the marine cyclopentadepsipeptide considered in this study [64]. The associated website allows the estimation of the most probable macromolecular targets of a small molecule, assumed as bioactive.

Pharmacokinetics is the process associated with the knowledge of the possible fate of a therapeutic compound in the organism which is very important knowledge within the process of development of a new drug. This has been usually done by analyzing the associated effects through individual indices that are called absorption, distribution, metabolism, excretion, and toxicity (ADMET) parameters. In this research, some ADMET parameters were estimated with the aid of Chemicalize and the SwissADME software available online [35]. Additional information about the 
pharmacokinetics parameters and the ADMET properties were obtained by resorting to pkCSM [36], a software application for the prediction of small-molecule pharmacokinetic properties using SMILES that can be accessed through its associated web page (https://biosig.unimelb.edu. $\mathrm{au} / \mathrm{pkcsm} /)$ (accessed March 2021).

\section{Results and Discussion}

The starting molecular structure of the marine cyclopentadepsipeptide to be studied was obtained from ChemSpider (https://www.chemspider.com), which is a chemical structure database available online that provides fast structure search access to millions of structures from many data sources, with information related to physical, chemical, and biological properties, interactive spectra, and literature references. A graphical sketch of the molecular structure of alternaramide is shown in Figure 1.

\subsection{Names, Identifiers, and Physicochemical Properties.} The names, identifiers, and basic properties of the alternaramide marine cyclopentadepsipeptide are presented in Table 1 while its geometrical, structural, and physicochemical properties are given in Table 2 .

This information could it be of interest for future QSAR studies based on the peptide as well as for potential derivatives designed for therapeutical purposes using peptidomimetics.

3.2. Conceptual DFT Calculations. The optimized molecular structure of alternaramide marine cyclopentadepsipeptide calculated according to the procedure presented in the Materials and Methods section is shown in Figure 2, while Figure 3 shows a schematic representation of the cyclopeptide showing an internal $\mathrm{H}$-bond formed between $\mathrm{O}(5)$ and the $\mathrm{H}$ atom attached to $\mathrm{N}(9)$.

It has been proved in our previous research on peptides [12-20] that the MN12SX density functional presents a Koopmans-compliant behavior. However, we consider that it is worth to perform further validation for the marine cyclopentadepsipeptide under study. This determination has been done by considering the in-house developed CDFT software tool including a comparison with the results that can be obtained using other density functionals. It has been shown for small molecules that long-range corrected density functionals are the best for reproducing the behavior prescribed by Janak's theorem [65] and its corollary, the ionization energy theorem. A recent study [66] has compared this behavior for a group of density functionals that includes the usual B3LYP [67-69] and PBE0 [70, 71] density functionals, the local density functionals BLYP $[68,69,72,73]$ and $\mathrm{PBE}[74]$ together with their long-range corrected counterparts, LC-BLYP and LC-PBE [75], three modern long-range corrected density functionals, CAM-B3LYP [76], LC- $\omega$ HPBE [77], and $\omega$ B97XD [78], and three recently proposed variants of the PBE0 density functional, RSX-PBE, RSX-PBE0, and RSX-PBE0-1/3 [79]. For the sake of completeness, we are presenting in Table 3 a comparison of the

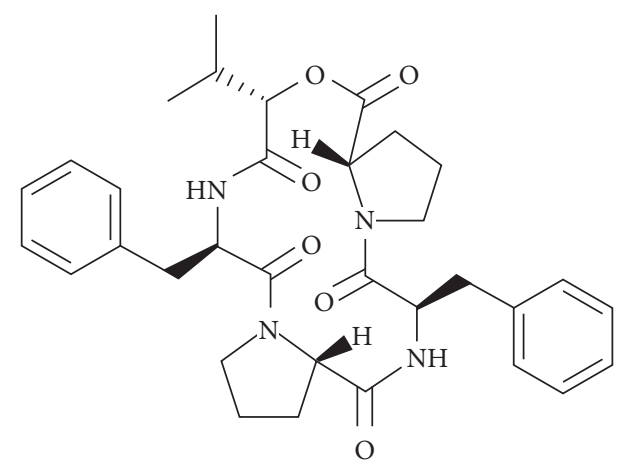

FIgURE 1: Graphical sketch of the molecular structure of the alternaramide marine cyclopentadepsipeptide.

fulfilment of the ionization energy theorem between the mentioned density functionals and the screened-exchange MN12SX density functional that has been considered in this and previous research for the study of peptides.

According to a recent study [66], the precise vertical ionization energy (VIE) may be obtained using both the SCF approach or the HOMO energy if one possesses the exact exchange-correlation functional. It can be derived from this assertion that a given approximate density functional will be more closer to the exact one as the values of the VIE that it renders from both methodologies will not differ. This is precisely what the KID procedure allows to verify for every tested density functional. From Table 3, the values for the KID descriptors are all very close to zero meaning that the MN12SX density functional has a Koopmans-compliant behavior (or the fulfilment of the Janak and ionization energy theorems) further justifying that the MN12SX/Def2TZVP/H2O is a model chemistry very adequate for the purpose of this research. Although some other density functionals have been shown to approximately fullfil those theorems for the case of small molecules, the results from this and previous research have demonstrated that this not the case for peptides and cyclopeptides [12-20]. The MN12SX density if the only one that allows the verification of these theorems not only for the case of the VIE but also for the vertical electron affinity thus rendering very accurate HOMO-LUMO gaps.

The values of the defined global reactivity descriptors (including the nucleophilicity $N$ ) for the alternaramide marine cyclopentadepsipeptide obtained using the mentioned CDFT tool are given in Table 4.

The global descriptors are an indication of the chemical reactivity of each molecule as a whole, and due to this, local reactivity descriptors have been designed for an estimation of the differences in the chemical reactivity between the regions within the molecule. The nucleophilic and electrophilic Fukui functions (NFF and EFF) [21-23] and the dual descriptor (DD) [80-85] are some of these local reactivity descriptors. They have been defined as follows: $\mathrm{NFF}=\rho_{N+1}(r)-\rho_{N}(r), \mathrm{EFF}=\rho_{N}(r)-\rho_{N-1}(r)$, and $\mathrm{DD}=$ $\Delta f(r)=(\partial f(r) / \partial N)_{v(r)}$, establishing connections between the electronic densities of different species as well as between the NFF and EFF. 
TABLE 1: Names, identifiers, and basic properties of the studied molecular system.

\begin{tabular}{|c|c|}
\hline Property & Value \\
\hline Common name & Alternaramide \\
\hline PubChem CID & 44605719 \\
\hline Molar mass & $588.705 \mathrm{~g} / \mathrm{mol}$ \\
\hline Exact mass & $588.294785024 \mathrm{Da}$ \\
\hline Formula & $\mathrm{C}_{33} \mathrm{H}_{40} \mathrm{~N}_{4} \mathrm{O}_{6}$ \\
\hline Composition & C (67.33\%), H (6.85\%), N (9.52\%), O (16.31\%) \\
\hline IUPAC name & $\begin{array}{l}\text { (3R,6S,12R,15S,18S)-3,12-Dibenzyl-15-(propan-2-yl)-16-oxa- } \\
\left.\text { 1,4,10,13-Tetraazatricyclo[16.3.0.0 } 0^{6,10}\right] \text { henicosane-2,5,11,14, }\end{array}$ \\
\hline & CC(C)[C@@ 17 -Pentone \\
\hline SMILES & $\begin{aligned}(\mathrm{CC} 2=\mathrm{CC}=\mathrm{CC}= & \mathrm{C} 2) \mathrm{NC}(=\mathrm{O})[\mathrm{C} @ @ \mathrm{H}] 2 \mathrm{CCCN} 2 \mathrm{C}(=\mathrm{O})[\mathrm{C} @ @ \mathrm{H}] \\
& (\mathrm{CC} 2=\mathrm{CC}=\mathrm{CC}=\mathrm{C} 2) \mathrm{NC} 1=\mathrm{O}\end{aligned}$ \\
\hline InChIKey & IZCWSRIIMBIBGB-MASCHLQQSA-N \\
\hline
\end{tabular}

TABle 2: Geometrical, structural, and physicochemical properties of the studied molecular system.

\begin{tabular}{lc}
\hline Property & Value \\
\hline Atom count & 83 \\
Non-hydrogen atom count & 43 \\
Asymmetric atom count & 5 \\
Rotatable atom count & 5 \\
Ring count & 5 \\
Aromatic ring count & 2 \\
Hetero ring count & 3 \\
FSP3 & 0.48 \\
Hydrogen bond donor count & 2 \\
Hydrogen bond acceptor count & 5 \\
Formal charge & 0 \\
van der Waals volume $\left(\AA^{3}\right)$ & 546.61 \\
van der Waals surface area $\left(\AA^{2}\right)$ & 870.17 \\
Solvent accessible surface area $\left(\AA^{2}\right)$ & 769.98 \\
Topological polar surface area $\left(\AA^{2}\right)$ & 125.12 \\
Polarizability $\left(\AA^{3}\right)$ & 62.04 \\
Molar refractivity $\left(\mathrm{cm}^{3} / \mathrm{mol}\right)$ & 158.15 \\
\hline
\end{tabular}

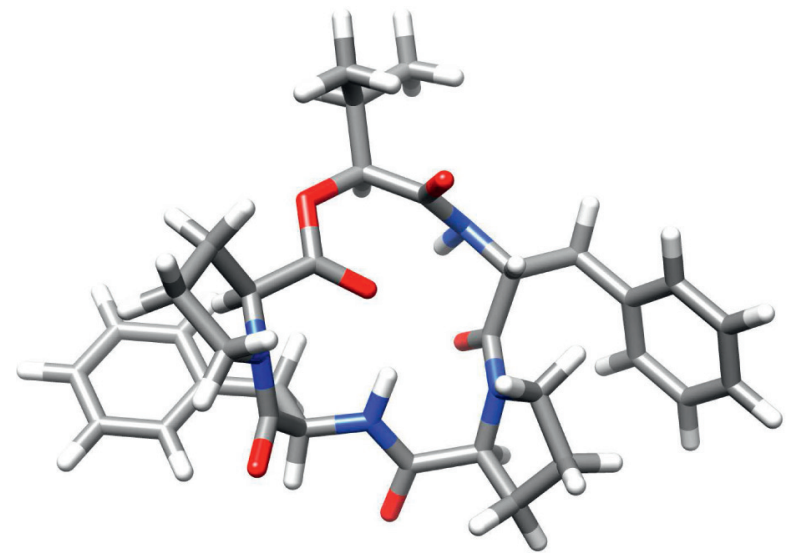

FIGURE 2: Optimized molecular structure of alternaramide.

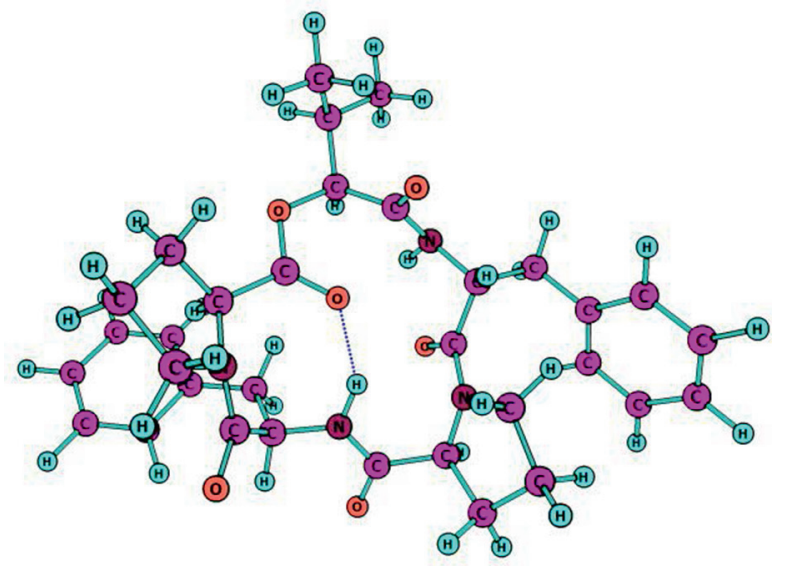

FIgURE 3: Molecular structure of alternaramide showing the internal H-bond.

TABLe 3: HOMO, LUMO, and SOMO energies, HOMO-LUMO gap, and the KID descriptors (all in $\mathrm{eV}$ ) for the alternaramide marine cyclopentadepsipeptide.

\begin{tabular}{lcccccccc}
\hline DF & HOMO & LUMO & SOMO & $\begin{array}{c}\mathrm{HL} \\
\text { gap }\end{array}$ & $\mathrm{J}_{I}$ & $\mathrm{~J}_{A}$ & $\mathrm{~J}_{H L}$ & $\Delta \mathrm{SL}$ \\
\hline BLYP & -5.31 & -1.42 & -0.78 & 3.89 & 0.29 & 0.41 & 0.50 & 0.63 \\
PBE & -5.51 & -1.62 & -1.02 & 3.89 & 0.29 & 0.38 & 0.48 & 0.60 \\
B3LYP & -6.63 & -0.80 & -1.12 & 5.83 & 0.21 & 0.16 & 0.26 & 0.32 \\
PBE0 & -6.93 & -0.63 & -1.33 & 6.30 & 0.35 & 0.36 & 0.51 & 0.70 \\
LC-BLYP & -9.52 & 1.92 & -2.48 & 11.44 & 2.76 & 2.18 & 3.52 & 4.40 \\
LC-PBE & -9.78 & 1.68 & -2.75 & 11.47 & 2.77 & 2.20 & 3.54 & 4.43 \\
CAM- & -8.11 & 0.64 & -2.48 & 8.75 & 1.47 & 1.55 & 2.14 & 3.12 \\
B3LYP & -9.47 & 1.72 & -2.59 & 11.19 & 2.64 & 2.14 & 3.40 & 4.31 \\
LC- $\omega$ HPBE & -9.11 & 0.64 & -2.48 & 8.75 & 1.47 & 1.55 & 2.14 & 3.12 \\
$\omega$ B97XD & -8.11 & -2.74 & 11.38 & 2.72 & 2.18 & 3.48 & 4.39 \\
RSX-PBE & -9.73 & 1.65 & -2.74 & \\
RSX-PBE0 & -9.73 & 1.70 & -2.73 & 11.43 & 2.77 & 2.19 & 3.54 & 4.43 \\
RSX-PBE0- & -9.74 & 1.72 & -2.73 & 11.46 & 2.80 & 2.20 & 3.57 & 4.45 \\
1/3 & -6.72 & -1.03 & -1.02 & 5.69 & 0.01 & 0.01 & 0.01 & 0.02 \\
MN12SX & -6.02 &
\end{tabular}


TABLE 4: Global reactivity descriptors for the alternaramide marine cyclopentadepsipeptide (all in $\mathrm{eV}$ ).

\begin{tabular}{lc}
\hline Descriptor & Value \\
\hline Electronegativity $\chi$ & 3.8787 \\
Hardness $\eta$ & 5.6894 \\
Electrophilicity $\omega$ & 1.3222 \\
Softness $S$ & 0.1751 \\
Nucleophilicity $N$ & 2.3978 \\
Electrodonating power $\omega^{-}$ & 4.9392 \\
Electroaccepting power $\omega^{+}$ & 1.0605 \\
Net electrophilicity $\Delta \omega^{ \pm}$ & 5.9998 \\
\hline
\end{tabular}

The NFF determines the sites of a molecular that are more susceptible to nucleophilic attacks while the EFF is an indication of regions that are more susceptible to electrophilic attacks. These local reactivity descriptors have been successfully used for the identification of the reactive sites. However, it has been found that the dual descriptor $\Delta f(r)$ or DD can describe simultaneously the nucleophilic and electrophilic sites within a molecule without ambiguities [85]. A graphical sketch of the dual descriptor (DD) for the alternaramide marine cyclopentadepsipeptide is presented in Figure 4 showing the regions where $\mathrm{DD}>0$ and $\mathrm{DD}<0$ for a better understanding of the local chemical reactivity of these molecules.

Table 5 presents a comparison of several reactivity descriptors: condensed electrophilicity $\omega_{k}$, condensed nucleophilicity $N_{k}$, and condensed dual descriptor $\Delta f_{k}$, over selected atoms of the alternaramide cyclopentadepsipeptide in relation with the molecular structure shown in Figure 5.

3.3. Chemoinformatics and Bioactivities. A compact representation of the parameters related to bioavailability can be displayed in a graphic way through the so called bioavailability radar shown in Figure 6 for the alternaramide marine cyclopentadepsipeptide.

It can be appreciated that the only problem for the alternaramide marine cyclopentadepsipeptide to be considered as therapeutic drug of easy availability is related to their size which is a bit greater than the ideal one.

The bioactivity score, that is, the measure of the ability of a given molecule to behave or interact with different receptors, for the alternaramide marine cyclopentadepsipeptide is presented in Table 6, while a graphical representation is shown in Figure 7, as the corresponding biological targets.

A chemical with a bioactivity score more than 0 is predicted to have significant biological activities, while values between -0.50 and 0.00 are moderately active. The molecular system is considered inactive if the bioactivity score is less than -0.50 . The findings clearly show that drug complexes' physiological activities may be mediated by many pathways, including interactions with GPCR ligands, protease inhibitors, and other enzymes. These bioactivity scores from Table 6 and Figure 6 suggest considerable interaction of the alternaramide cyclopeptide with GPCR ligands and protease inhibitors, while showing moderate interactions with the other receptors.

The pharmacokinetics of a drug are assessed in an ADMET research, which stands for absorption, distribution, metabolism, excretion, and toxicity. Predicting a medication's fate and effects inside the body, such as how much of a drug is absorbed if taken orally versus how much is absorbed in the gastrointestinal tract, is an essential element of drug development. Similarly, if absorption is poor, the distribution and metabolism of the drug would be altered, potentially leading to neurotoxicity and nephrotoxicity. The goal of the research is to figure out how a drug molecule behaves within an organism. As a result, one of the most important aspects of computational drug design is an ADMET analysis.

If a substance is injected into the bloodstream, it can reach a tissue. A medicine is typically delivered through mucous surfaces such as the digestive tract, i.e., intestinal absorption, before being taken up by target cells. Poor substance solubility, difficulty to permeate the intestinal wall, and chemical instability in the stomach are all factors that contribute to drug absorption being reduced following oral delivery. Absorption is crucial since it influences a compound's bioavailability. Oral delivery, such as inhalation or intravenously, is less desirable for drugs with limited absorption [36, 86].

The computed absorption properties of the alternaramide marine cyclopentadepsipeptide are presented in Table 7.

A given compound is considered to have a high Caco-2 permeability through the human intestinal mucosa for predicted values $>0.90$, presenting the alternaramide marine cyclopentadepsipeptide a value similar to the ideal one. The intestine is normally the primary site for absorption of a drug from an orally administered solution. The intestinal absorption predicts the percentage of a compound to be absorbed through the human intestine considering an absorbance of less than $30 \%$ to be poorly absorbed. From Table 7, the alternaramide marine cyclopentadepsipeptide is predicted to be well absorbed. Toxins and xenobiotics are extruded from cells by the P-glycoprotein, which acts as a biological barrier. The model predicts whether or not a given substance will be a P-glycoprotein substrate. The alternaramide marine cyclic pentadepsipeptide has a promising future. Modulation of P-glycoprotein-mediated transport has substantial pharmacokinetic implications for P-glycoprotein substrates, which can be used for specific therapeutic benefits or cause contraindications. Thus, this study predicts that the alternaramide marine cyclopentadepsipeptide considered in this study will not act as P-glycoprotein I and II inhibitors. It is also possible to forecast if a certain substance will be skin permeable. If a compound's $\log \mathrm{Kp}$ is more than -2.5 , it is said to have low skin permeability. It suggests that alternaramide may be useful in the development of transdermal medication delivery systems [36].

The computed distribution properties of the alternaramide marine cyclopentadepsipeptide are presented in Table 8.

The total dose of a drug requires a volume to be uniform in blood plasma which is named VDss. The drug will be more distributed in the tissue rather than in the plasma for higher VDss. From Table 8, a low value of VDss is found for the alternaramide marine cyclopentadepsipeptide. The efficacy of a given drug may be affected by the degree to which 


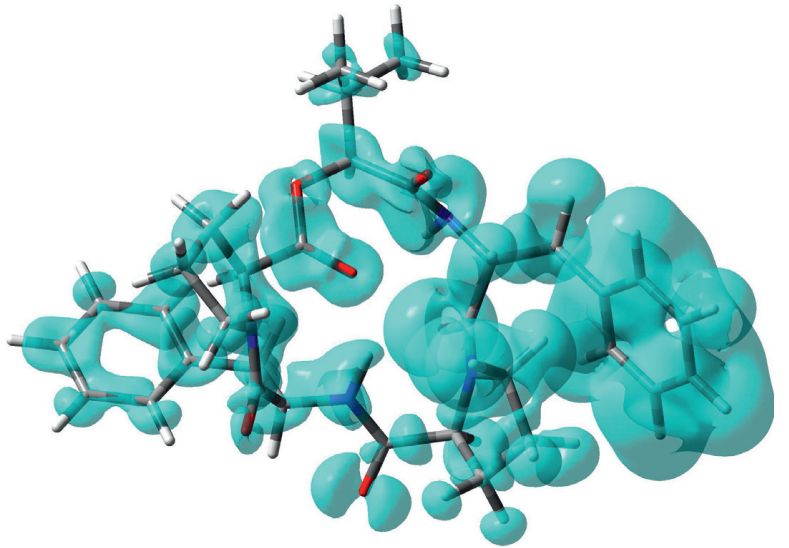

(a)

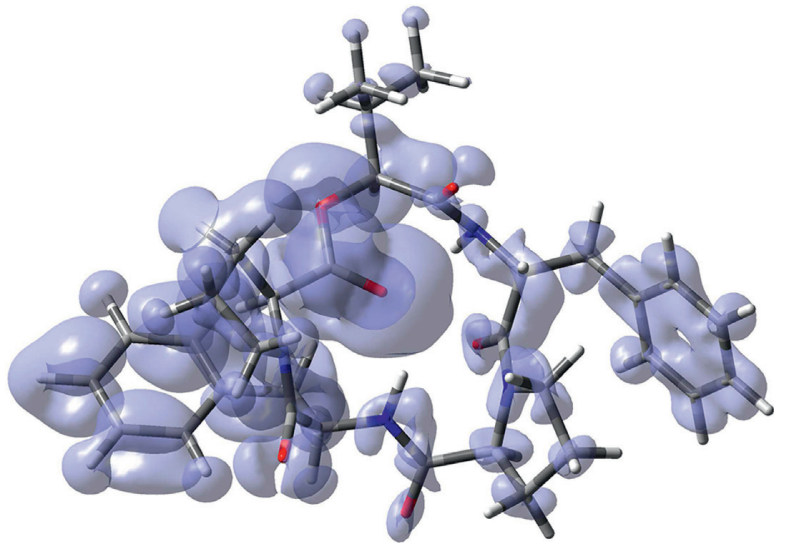

(b)

FIgURE 4: Graphical representation of the dual descriptor (DD) of the alternaramide marine cyclopentadepsipeptide. (a) DD $>0$. (b) $\mathrm{DD}<0$.

TABLE 5: Comparison of several reactivity descriptors: condensed electrophilicity $\omega_{k}$, condensed nucleophilicity $N_{k}$, and condensed dual descriptor $\Delta f_{k}$, over selected atoms of the alternaramide cyclopentadepsipeptide ( $\mathrm{H}$ atoms are not shown).

\begin{tabular}{|c|c|c|c|}
\hline \\
\hline \multicolumn{4}{|c|}{$\begin{array}{l}\text { Alternaramide } \\
\text { Atom }\end{array}$} \\
\hline $1(\mathrm{O})$ & 0.0828 & 0.0087 & 0.0591 \\
\hline $2(\mathrm{O})$ & 0.0143 & 0.0612 & -0.0146 \\
\hline $3(\mathrm{O})$ & 0.0315 & 0.0120 & 0.0189 \\
\hline $4(\mathrm{O})$ & 0.0134 & 0.1182 & -0.0391 \\
\hline $5(\mathrm{O})$ & 0.1673 & 0.0033 & 0.1255 \\
\hline $6(\mathrm{O})$ & 0.0364 & 0.0490 & 0.0072 \\
\hline $7(\mathrm{~N})$ & 0.0035 & 0.0957 & 0.0373 \\
\hline $8(\mathrm{~N})$ & 0.0057 & 0.0061 & 0.0018 \\
\hline $9(\mathrm{~N})$ & 0.0036 & 0.0169 & -0.0043 \\
\hline $10(\mathrm{~N})$ & 0.0130 & 0.0596 & -0.0158 \\
\hline C (11) & 0.0025 & 0.0212 & -0.0069 \\
\hline C (12) & 0.0019 & 0.0160 & -0.0053 \\
\hline C (13) & 0.0358 & 0.0028 & 0.0259 \\
\hline C (14) & 0.0016 & 0.0276 & -0.0103 \\
\hline C (15) & 0.0015 & 0.0507 & -0.0200 \\
\hline C (16) & 0.0212 & 0.0017 & 0.0154 \\
\hline C (17) & 0.0076 & 0.0022 & 0.0049 \\
\hline C (18) & 0.0098 & 0.0016 & 0.0067 \\
\hline C (19) & 0.0022 & 0.0052 & -0.0006 \\
\hline C (20) & 0.0094 & 0.0218 & -0.0020 \\
\hline C (21) & 00158 & 0.0037 & 0.0105 \\
\hline C (22) & 0.0067 & 0.0306 & -0.0077 \\
\hline C (23) & 0.0041 & 0.0341 & -0.0111 \\
\hline C (24) & 0.2202 & 0.0063 & 0.1644 \\
\hline C (25) & 0.0098 & 0.0025 & 0.0064 \\
\hline C (26) & 0.0172 & 0.0064 & 0.0103 \\
\hline C (27) & 0.0031 & 0.0047 & -0.0173 \\
\hline C (28) & 0.0053 & 0.0035 & 0.0025 \\
\hline C (29) & 0.0130 & 0.0205 & 0.0013 \\
\hline C (30) & 0.0223 & 0.0007 & 0.0166 \\
\hline C (31) & 0.0001 & 0.1484 & -0.0618 \\
\hline C (32) & 0.0042 & 0.0028 & 0.0020 \\
\hline C (33) & 0.0051 & 0.0018 & 0.0031 \\
\hline C (34) & 0.0326 & 0.0018 & 0.0240 \\
\hline C (35) & 0.0236 & 0.0012 & 0.0174 \\
\hline
\end{tabular}


TABle 5: Continued.

\begin{tabular}{lccc}
\hline $\begin{array}{l}\text { Alternaramide } \\
\text { Atom }\end{array}$ & $\omega_{k}$ & $N_{k}$ & $\Delta f_{k}$ \\
\hline C (36) & 0.0010 & 0.0640 & -0.0260 \\
C (37) & 0.0015 & 0.1879 & -0.0772 \\
C (38) & 0.0227 & 0.0015 & 0.0166 \\
C (39) & 0.0289 & 0.0020 & 0.0211 \\
C (40) & 0.0015 & 0.1644 & -0.0675 \\
C (41) & 0.0015 & 0.0024 & -0.0321 \\
C (42) & 0.0468 & 0.0024 & 0.0345 \\
C $(43)$ & 0.0017 & 0.2123 & -0.0873 \\
\hline
\end{tabular}

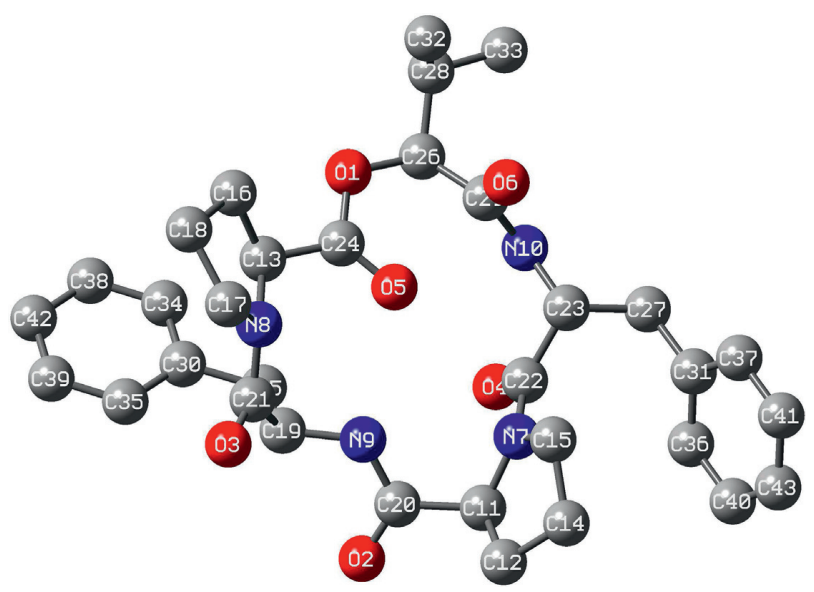

FIgURE 5: Molecular structure of alternaramide showing the labelling over the atoms. $\mathrm{H}$ atoms are not shown.

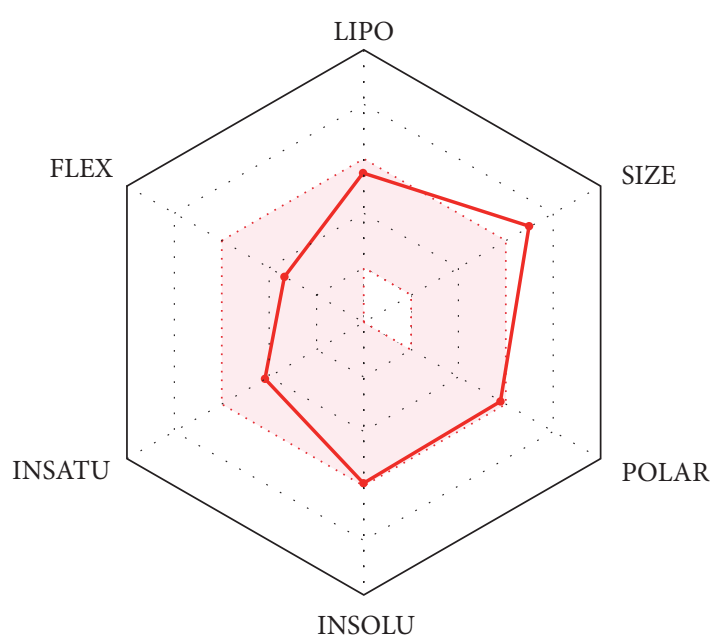

FIGURE 6: Bioactivity radar of the alternaramide marine cyclopentadepsipeptide.

it binds proteins within blood. The fraction unbound forecasts the fraction of plasma that will be unbound, giving the value in Table 8 . The ability of a drug to cross into the brain is an important descriptor to be known because it can help to reduce side effects and toxicities and is measured using the blood-brain permeability parameter. A $\log B B$
TABLE 6: Bioactivity scores of the alternaramide marine cyclopentadepsipeptide calculated for its biological target interactions.

\begin{tabular}{lc}
\hline Property & Value \\
\hline GPCR ligand & 0.16 \\
Ion channel modulator & -0.49 \\
Nuclear receptor ligand & -0.40 \\
Kinase inhibitor & -0.35 \\
Protease inhibitor & 0.51 \\
Enzyme inhibitor & -0.17 \\
\hline
\end{tabular}

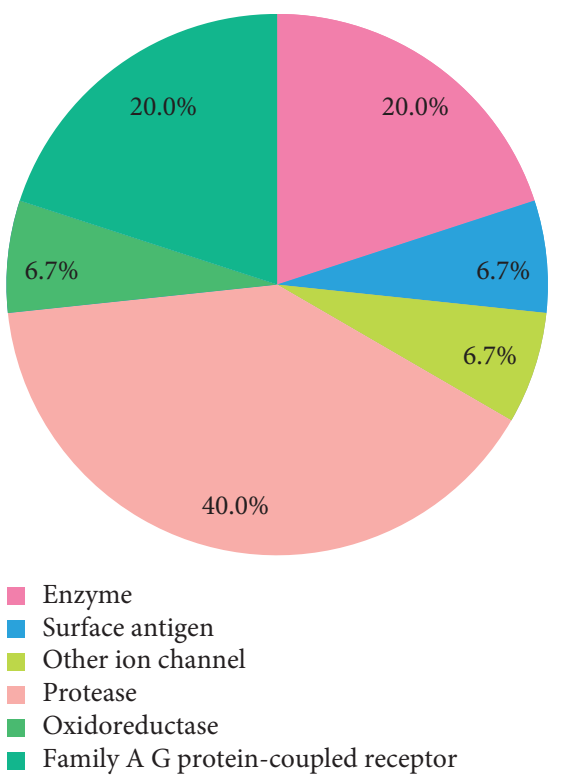

FIGURE 7: Predicted biological targets of alternaramide marine cyclopentadepsipeptide.

TABLE 7: Absorption properties of the alternaramide marine cyclopentadepsipeptide.

\begin{tabular}{lc}
\hline Property & Value \\
\hline Water solubility & -4.518 \\
Caco-2 permeability & 0.907 \\
Intestinal absorption & 74.153 \\
Skin permeability & -2.737 \\
P-glycoprotein substrate & + \\
P-glycoprotein I inhibitor & - \\
P-glycoprotein II inhibitor & - \\
\hline
\end{tabular}


TABLE 8: Distribution properties of the alternaramide marine cyclopentadepsipeptide.

\begin{tabular}{lc}
\hline Property & Value \\
\hline VDss & -0.141 \\
Fraction unbound & 0.016 \\
BBB permeability & -0.610 \\
CNS permeability & -2.971 \\
\hline
\end{tabular}

score of $>-0.3$ indicates that a possible therapeutic treatment will easily penetrate the blood-brain barrier, whereas a $\log B \mathrm{~B}$ value of $>-1$ indicates that the medicine will be poorly dispersed to the brain. Another measurement is the CNS permeability with a value of -2.971 predicted for the alternaramide marine cyclopentadepsipeptide meaning that this drug will be unable to penetrate the central nervous system (CNS) [36].

The computed metabolism properties of the alternaramide marine cyclopentadepsipeptide are presented in Table 9.

Because it oxidizes xenobiotics to promote excretion, cytochrome P450 is a key detoxification enzyme in the body, primarily found in the liver [36]. As can be seen from Table 6 , the alternaramide marine cyclopentadepsipeptide is predicted as not being an inhibitor for all the P450 cytochrome isoforms with the exception of CYP3A4. It is also crucial to know whether or not a medicine is a cytochrome P450 substrate. According to the forecast, this will not be the case for CYP2D6, but it will be for CYP3A4.

The computed excretion properties of the alternaramide marine cyclopentadepsipeptide are presented in Table 10.

Drug clearance occurs as a combination of hepatic clearance and renal clearance, and it is related to bioavailability, thus important for determining dosing rates. The predicted total clearance of the alternaramide marine cyclopentadepsipeptide is given in $\log (\mathrm{ml} / \mathrm{min} / \mathrm{kg})$. OCT2 is a renal uptake transporter that plays a key role in drug disposition and clearance in the kidneys. The cyclopentadepsipeptide used in this investigation is unlikely to act as an OCT2 substrate [36].

The computed toxicity properties of the alternaramide marine cyclopentadepsipeptide are presented in Table 11.

The AMES toxicity test uses bacteria to check a compound's mutagenesis potential. A positive test indicates that the substance is mutagenic and so could cause cancer. The predictions are negative for the cyclopentadepsipeptide under study. The maximum recommended tolerated dose (MRTD) provides an estimate of the toxic dose threshold of chemicals in humans. The MRTD is low for the alternaramide marine cyclopentadepsipeptide. The inhibition of the potassium channels encoded by hERG is the principal cause for the development of acquiring long QT syndrome, thus leading to fatal ventricular arrhythmia. The predictions indicate that alternaramide cyclopentadepsipeptide is unlikely to be a hERG I inhibitor, but it is the opposite for hERG II. The lethal dosage values (LD50) are a common measure of acute toxicity, and they are defined as the amount of a substance that kills $50 \%$ of a set of test animals. The ORAT (oral rat acute toxicity) and ORCT (oral rat
TABLE 9: Metabolism properties of the alternaramide marine cyclopentadepsipeptide.

\begin{tabular}{lc}
\hline Property & Value \\
\hline CYP2D6 substrate & - \\
CYP3A4 substrate & + \\
CYP1A2 inhibitor & - \\
CYP2C19 inhibitor & - \\
CYP2C9 inhibitor & - \\
CYP2D6 inhibitor & - \\
CYP3A4 inhibitor & + \\
\hline
\end{tabular}

TABLE 10: Excretion properties of the alternaramide marine cyclopentadepsipeptide.

\begin{tabular}{lc}
\hline Property & Value \\
\hline Total clearance & 0.670 \\
Renal OCT2 substrate & - \\
\hline
\end{tabular}

TABLE 11: Toxicity properties of the alternaramide marine cyclopentadepsipeptide.

\begin{tabular}{lc}
\hline Property & Value \\
\hline AMES toxicity & - \\
MRTD & -0.442 \\
hERG I inhibition & - \\
hERG II inhibition & + \\
ORAT & 3.665 \\
ORCT & 2.890 \\
Hepatotoxicity & + \\
Skin sensitisation & - \\
T. pyriformis toxicity & 0.285 \\
\hline
\end{tabular}

chronic toxicity) metrics can be used to assess this. Druginduced liver injury is a prominent source of medication attrition and a critical safety concern for drug development. As a result, hepatoxicity is linked to a disruption in the liver's normal function, and alternaramide has a favorable prognosis. Skin sensitisation, on the other hand, has a negative outlook. T. pyriformis is a protozoan bacterium whose toxicity is frequently employed as a hazardous endpoint. Toxicity is defined as a predicted value of $>-0.5$ for a given chemical [36].

\section{Conclusion}

Through our proposed computational peptidology methodology, the alternaramide marine cyclopentadepsipeptide isolated from marine sources was studied using some techniques commonly used in the process of drug discovery and development, demonstrating that this peptide can be considered a potential therapeutic drug.

Some useful descriptors for future QSAR studies, the physicochemical properties, its biological targets, and the ADMET (absorption, distribution, metabolism, excretion, and toxicity) indices related to the bioavailability and pharmacokinetics of these marine cyclopentadepsipeptide under study were predicted and analyzed. 
With this knowledge in mind, the chemical reactivity of the studied alternaramide marine cyclopentadepsipeptide has been exhaustively analyzed through the optimization of their structures using the DFTBA methodology and the calculation of their electronic properties with the consideration of a best quality model chemistry, that is, MN12SX/ Def2TZVP/H20, which has been already used in previous research for the study of cyclic peptides, showing its usefulness for this kind of calculations being complemented with the calculation of the conceptual DFT global and local reactivity descriptors.

\section{Data Availability}

The data used to support the findings of this study are available from the corresponding author upon request.

\section{Conflicts of Interest}

The authors declare that they have no conflicts of interest.

\section{Authors' Contributions}

$\mathrm{NFH}$ and JF were responsible for research and data analysis. DGM was responsible for research, data analysis, and writing the manuscript.

\section{Acknowledgments}

NFH and DGM are researchers of CIMAV and CONACYT and would like to thank both institutions for partial support.

\section{References}

[1] F. Sarabia, S. Chammaa, A. Ruiz, L. Ortiz, and F. Herrera, "Chemistry and biology of cyclic depsipeptides of medicinal and biological interest," Current Medicinal Chemistry, vol. 11, no. 10, pp. 1309-1332, 2004.

[2] G. S. Bagavananthem Andavan and R. Lemmens-Gruber, "Cyclodepsipeptides from marine sponges: natural agents for drug research," Marine Drugs, vol. 8, no. 3, pp. 810-834, 2010.

[3] D. Newman and G. Cragg, "Marine-sourced anti-cancer and cancer pain control agents in clinical and late preclinical development," Marine Drugs, vol. 12, no. 1, pp. 255-278, 2014.

[4] S. Sivanathan and J. . Scherkenbeck, "Cyclodepsipeptides: a rich source of biologically active compounds for drug research," Molecules, vol. 19, no. 8, pp. 12368-12420, 2014.

[5] M. Mehbub, J. Lei, C. Franco, and W. Zhang, "Marine sponge derived natural products between 2001 and 2010: trends and opportunities for discovery of bioactives," Marine Drugs, vol. 12, no. 8, pp. 4539-4577, 2014.

[6] J. Kitagaki, G. Shi, S. Miyauchi, S. Murakami, and Y. Yang, "Cyclic depsipeptides as potential cancer therapeutics," AntiCancer Drugs, vol. 26, no. 3, pp. 259-271, 2015.

[7] M. Pelay-Gimeno, J. Tulla-Puche, and F. Albericio, "“Headto-Side-Chain" cyclodepsipeptides of marine origin," Marine Drugs, vol. 11, no. 5, pp. 1693-1717, 2013.

[8] M. Pelay-Gimeno, F. Albericio, and J. Tulla-Puche, "Synthesis of complex head-to-side-chain cyclodepsipeptides," Nature Protocols, vol. 11, no. 10, pp. 1924-1947, 2016.

[9] M.-Y. Kim, J. H. Sohn, J. S. Ahn, and H. Oh, “Alternaramide, a cyclic depsipeptide from the marine-derived fungus
Alternaria sp.SF-5016," Journal of Natural Products, vol. 72, no. 11, pp. 2065-2068, 2009.

[10] W. Ko, J. H. Sohn, J.-H. Jang et al., "Inhibitory effects of Alternaramide on inflammatory mediator expression through TLR4-MyD88-mediated inhibition of NF- $\kappa$ B and MAPK pathway signaling in lipopolysaccharide-stimulated RAW264.7 and BV2 cells," Chemico-Biological Interactions, vol. 244, pp. 16-26, 2016.

[11] A. Horton, O. May, M. Elsegood, and M. Kimber, "Total synthesis of the marine-derived cyclic depsipeptide Alternaramide," Synlett, vol. 2011, no. 06, pp. 797-800, 2011.

[12] J. Frau, N. Flores-Holguín, and D. Glossman-Mitnik, "Chemical reactivity properties, pKa values, AGEs inhibitor abilities and bioactivity scores of the mirabamides A-H peptides of marine origin studied by means of conceptual DFT," Marine Drugs, vol. 16, no. 9, pp. 302-319, 2018.

[13] N. Flores-Holguín, J. Frau, and D. Glossman-Mitnik, "Chemical-reactivity properties, drug likeness, and bioactivity scores of Seragamides A-F anticancer marine peptides: conceptual density functional theory viewpoint," Computation, vol. 7, no. 3, 2019.

[14] J. Frau, N Flores-Holguín, and D Glossman-Mitnik, "Chemical reactivity theory and empirical bioactivity scores as computational Peptidology alternative tools for the study of two anticancer peptides of marine origin," Molecules, vol. 24, no. 6, p. 1115, 2019.

[15] N. Flores-Holguín, J. Frau, and D. Glossman-Mitnik, "Computational prediction of bioactivity scores and chemical reactivity properties of the parasin I therapeutic peptide of marine origin through the calculation of global and local conceptual DFT descriptors f," Theoretical Chemistry Accounts, vol. 138, no. 6, 2019.

[16] N. Flores-Holguín, J. Frau, and D. Glossman-Mitnik, "A fast and simple evaluation of the chemical reactivity properties of the pristinamycin family of antimicrobial peptides," Chemical Physics Letters, vol. 739, p. 137021, 2020.

[17] N. Flores-Holguín, J. Frau, and D. Glossman-Mitnik, "Conceptual DFT-based computational Peptidology of marine," Natural Compounds: Discodermins A-H”. Molecules, vol. 25, no. 18 , p. $4158,2020$.

[18] N. Flores-Holguín, J. Frau, and D. Glossman-Mitnik, "Virtual screening of marine natural compounds by means of chemoinformatics and CDFT-based computational Peptidology," Marine Drugs, vol. 18, no. 9, p. 478, 2020.

[19] N. Flores-Holguín, J. Frau, and D. Glossman-Mitnik, "Conceptual DFT as a helpful chemoinformatics tool for the study of the clavanin family of antimicrobial marine peptides," in Sergio Ricardo De Lazaro, Luis Henrique Da Silveira Lacerda and Renan Augusto Pontes Ribeiro, pp. 57-67, IntechOpen, London, UK, 2021, Density Functional Theory, chapter 3.

[20] N. Flores-Holguín, J. Frau, and D. Glossman-Mitnik, "In silico pharmacokinetics, ADMET study and conceptual DFT analysis of two plant cyclopeptides isolated from rosaceae as a computational Peptidology approach," Frontiers in Chemistry, vol. 9, Article ID 708364, 2021.

[21] R. G. Parr and W. Yang, Density-Functional Theory of Atoms and Molecules, Oxford University Press, New York, NY, USA, 1989.

[22] H. Chermette, "Chemical reactivity indexes in density functional theory," Journal of Computational Chemistry, vol. 20, no. 1, pp. 129-154, 1999.

[23] P. Geerlings, F. De Proft, and W. Langenaeker, "Conceptual density functional theory," Chemical Reviews, vol. 103, pp. 1793-1873, 2003. 
[24] J. L. Gázquez, A. Cedillo, and A. Vela, "Electrodonating and electroaccepting powers," Journal of Physical Chemistry A, vol. 111, no. 10, pp. 1966-1970, 2007.

[25] P. K. Chattaraj, A. Chakraborty, and S. Giri, "Net electrophilicity," Journal of Physical Chemistry A, vol. 113, no. 37, pp. 10068-10074, 2009.

[26] P. Geerlings, E. Chamorro, P. Kumar Chattaraj et al., "Conceptual density functional theory: status, prospects, issues," Theoretical Chemistry Accounts, vol. 139, no. 2, p. 36, 2020.

[27] T. Engel and J. Gasteiger, Eds., Applied Chemoinformatics: Achievements and Future Opportunities, Wiley VCH, Weinheim, Germany, 2018.

[28] T. Engel and J. Gasteiger, Eds., Chemoinformatics: Basic Concepts and Methods, Wiley VCH, Weinheim, Germany, 2018.

[29] J. . Bajorath, Ed., Chemoinformatics for Drug Discovery, WILEY, A John Wiley \& Sons Publication, New Jersey, NJ, USA, 2014.

[30] A. Varnek and A. Tropsha, Eds., Chemoinformatics Approaches to Virtual Screening, Royal Society of Chemistry, Cambridge, UK, 2008.

[31] R. Guha and A. Bender, Eds., Computational Approaches in Cheminformatics and Bioinformatics, Wiley, Hoboken, N.J, USA, 2012.

[32] B. Benjamin, Basic Principles of Drug Discovery and Development, Academic Press, Amsterdam Netherlands, 2015.

[33] J. L. Medina-Franco and F. I. Saldívar-González, "Cheminformatics to characterize pharmacologically," Active Natural Products. Biomolecules, vol. 10, no. 11, p. 11, 2020.

[34] B. F. Begam and J. S. Kumar, "A study on Cheminformatics and its applications on modern drug discovery," Procedia Engineering, vol. 38, pp. 1264-1275, 2012.

[35] A. Daina, O. Michielin, and V. Zoete, "SwissADME: a free web tool to evaluate pharmacokinetics, drug-likeness and medicinal chemistry friendliness of small molecules," Scientific Reports, vol. 7, no. 1, p. 42717, 2017.

[36] E. V. Douglas, T. L. B. Pires, and D. B. Ascher, "pkC. S. M.: Predicting small-molecule pharmacokinetic and toxicity properties using graph-based signatures," Journal of Medicinal Chemistry, vol. 58, no. 9, pp. 4066-4072, 2015.

[37] E. Lewars, Computational Chemistry - Introduction to the Theory and Applications of Molecular and Quantum Mechanics, Kluwer Academic Publishers, Dordrecht, Netherlands, 2003.

[38] D. C. Young, Computational Chemistry - A Practical Guide for Applying Techniques to Real-World Problems, John Wiley \& Sons, New York, NY, USA, 2001.

[39] F. Jensen, Introduction to Computational Chemistry, John Wiley \& Sons, Chichester, UK, 2nd edition, 2007.

[40] C. J. Cramer, Essentials of Computational Chemistry - Theories and Models, John Wiley \& Sons, Chichester, UK, 2nd edition, 2004.

[41] L. R. Domingo, E. Chamorro, and P. Perez, "Understanding the reactivity of captodative ethylenes in polar cycloaddition reactions. A theoretical study," Journal of Organic Chemistry, vol. 73, no. 12, pp. 4615-4624, 2008.

[42] P. Jaramillo, L. R. Domingo, E. Chamorro, and P. Pérez, “A further exploration of a nucleophilicity Index based on the gas-phase ionization potentials," Journal of Molecular Structure: THEOCHEM, vol. 865, no. 1-3, pp. 68-72, 2008.

[43] L. R. Domingo and J. A. Sáez, "Understanding the mechanism of polar diels-alder reactions," Organic and Biomolecular Chemistry, vol. 7, no. 17, pp. 3576-3583, 2009.
[44] R. Luis, "Domingo and patricia perez. "The nucleophilicity N Index in organic chemistry," Organic and Biomolecular Chemistry, vol. 9, pp. 7168-7175, 2011.

[45] R. Luis, "Domingo, mar ríos-gutiérrez and patricia pérez. "Applications of the conceptual density functional theory indices to organic chemistry reactivity," Molecules, vol. 21, p. 748, 2016.

[46] A. Thomas, "Merck molecular force field. I. Basis, form, scope, parameterization, and performance of MMFF94," Journal of Computational Chemistry, vol. 17, no. 5-6, pp. 490-519, 1996.

[47] A. Thomas, "Merck molecular force field. II. MMFF94 van der Waals and electrostatic parameters for intermolecular interactions," Journal of Computational Chemistry, vol. 17, no. 5-6, pp. 520-552, 1996.

[48] A. Thomas, "MMFF VI. MMFF94s option for energy minimization studies," Journal of Computational Chemistry, vol. 20, no. 7, pp. 720-729, 1999.

[49] A. H. Thomas and B. R. Thomas, "Merck molecular force field. IV. Conformational energies and geometries for MMFF94," Journal of Computational Chemistry, vol. 17, no. 5-6, pp. 587-615, 1996.

[50] A. Thomas, "Merck molecular force field. V. Extension of MMFF94 using experimental data, additional computational data, and empirical rules," Journal of Computational Chemistry, vol. 17, no. 5-6, pp. 616-641, 1996.

[51] M. J. Frisch, G. W. Trucks, H. B. Schlegel et al., Gaussian 16 Revision C.01, Gaussian Inc, Wallingford CT, UK, 2016.

[52] R. Peverati and D. G. Truhlar, "Screened-exchange density functionals with broad accuracy for chemistry and solid-state physics," Physical Chemistry Chemical Physics, vol. 14, no. 47, pp. 16187-16191, 2012.

[53] F. Weigend and R. Ahlrichs, "Balanced basis sets of split valence, triple zeta valence and quadruple zeta valence quality for $\mathrm{H}$ to Rn: design and assessment of accuracy," Physical Chemistry Chemical Physics, vol. 7, no. 18, pp. 3297-3305, 2005.

[54] F. Weigend, "Accurate Coulomb-fitting basis sets for $\mathrm{H}$ to Rn," Physical Chemistry Chemical Physics, vol. 8, no. 9, pp. 1057-1065, 2006.

[55] N. Flores-Holguín, J. Frau, and D. Glossman-Mitnik, "Computational Peptidology assisted by conceptual density functional theory for the study of five new antifungal tripeptides,” ACS Omega, vol. 4, no. 7, pp. 12555-12560, 2019.

[56] N. Flores-Holguín, J. Frau, and D. Glossman-Mitnik, "Conceptual DFT as a helpful chemoinformatics tool for the study of the clavanin family of antimicrobial marine peptides," in Density Functional Theory Calculations, pp. 1-11, IntechOpen, Rijetia, 2019.

[57] J. Frau and D. Glossman-Mitnik, "Molecular reactivity and absorption properties of melanoidin blue-G1 through conceptual DFT," Molecules, vol. 23, no. 3, pp. 559-615, 2018.

[58] J. Frau and D. Glossman-Mitnik, "Conceptual DFT study of the local chemical reactivity of the dilysyldipyrrolones A and B intermediate melanoidins," Theoretical Chemistry Accounts, vol. 137, no. 5, p. 1210, 2018.

[59] J. Frau and D. Glossman-Mitnik, "Conceptual DFT study of the local chemical reactivity of the colored BISARG melanoidin and its protonated derivative," Frontiers in Chemistry, vol. 6, no. 136, pp. 1-9, 2018.

[60] J. Frau and D. Glossman-Mitnik, "Computational study of the chemical reactivity of the blue-M1 intermediate melanoidin," Computational and Theoretical Chemistry, vol. 1134, pp. 2229, 2018. 
[61] J. Frau and D. Glossman-Mitnik, "Chemical reactivity theory applied to the calculation of the local reactivity descriptors of a colored maillard reaction product," Chemical Science International Journal, vol. 22, no. 4, pp. 1-14, 2018.

[62] J. Frau and D. Glossman-Mitnik, "Blue M2: an intermediate melanoidin studied via conceptual DFT," Journal of Molecular Modeling, vol. 24, no. 138, pp. 1-13, 2018.

[63] A. V. Marenich, C. J. Cramer, and D. G. Truhlar, "Universal solvation model based on solute Electron density and on a continuum model of the solvent defined by the bulk dielectric constant and atomic surface tensions," The Journal of Physical Chemistry B, vol. 113, no. 18, pp. 6378-6396, 2009.

[64] A. Daina, O. Michielin, and V. Zoete, "SwissTargetPrediction: updated data and new features for efficient prediction of protein targets of small molecules," Nucleic Acids Research, vol. 47, no. W1, p. W357, 2019.

[65] J. F. Janak, "Proof that $\partial \mathrm{E} \partial \mathrm{ni}=\varepsilon$ in density-functional theory," Physical Review B, vol. 18, no. 12, pp. 7165-7168, 1978.

[66] S. Kanchanakungwankul and G. Donald, "Examination of how well long-range-corrected density functionals satisfy the ionization energy theorem," Journal of Chemical Theory and Computation, vol. 17, no. 8, pp. 4823-4830, 2021.

[67] A. D. Becke, "Density-functional thermochemistry. III. The role of exact exchange," The Journal of Chemical Physics, vol. 98, no. 7, pp. 5648-5652, 1993.

[68] C. Lee, W. Yang, and R. G. Parr, "Development of the collesalvetti correlation-energy formula into a functional of the Electron density," Physical Review B, vol. 37, no. 2, pp. 785-789, 1988.

[69] S. H. Vosko, L. Wilk, and M. Nusair, "Accurate spin-dependent Electron liquid correlation energies for local spin density calculations: a critical analysis," Canadian Journal of Physics, vol. 58, no. 8, pp. 1200-1211, 1980.

[70] C. Adamo and V. Barone, "Toward reliable density functional methods without adjustable parameters: the PBE0 model," Chemical Physics, vol. 110, pp. 6158-6169, 1999.

[71] M. Ernzerhof and E. Gustavo, "Assessment of the perdewburke-ernzerhof exchange-correlation functional," The Journal of Chemical Physics, vol. 110, no. 11, pp. 5029-5036, 1999.

[72] A. D. Becke, "Density-functional exchange-energy approximation with correct asymptotic behavior," Physical Review A, vol. 38, no. 6, pp. 3098-3100, 1988.

[73] P. J. Stephens, F. J. Devlin, C. F. Frisch, and C. F. Chabalowski, "Ab initio calculation of vibrational absorption and circular dichroism spectra using density functional force fields," The Journal of Physical Chemistry, vol. 98, no. 45, pp. 1162311627, 1994.

[74] P. John, K. Burke, and M. Ernzerhof, "Generalized gradient approximation made simple," Physical Review Letters, vol. 77, pp. 3865-3868, 1996.

[75] H. Iikura, T. Takao, T. Yanai, and K. Hirao, "A long-range correction scheme for generalized-gradient-approximation exchange functionals," The Journal of Chemical Physics, vol. 115, no. 8, pp. 3540-3544, 2001.

[76] T. Yanai, D. P. Tew, and N. C. Handy, "A new hybrid exchange-correlation functional using the coulomb-attenuating method (CAM-B3LYP)," Chemical Physics Letters, vol. 393, no. 1-3, pp. 51-57, 2004.

[77] T. M. Henderson, A. F. Izmaylov, G. Scalmani, and G. E. Scuseria, "Can short-range hybrids describe long-rangedependent properties?" The Journal of Chemical Physics, vol. 131, no. 4, Article ID 044108, 2009.

[78] J.-D. Chai and M. Head-Gordon, "Long-range corrected hybrid density functionals with damped atom-atom dispersion corrections," Physical Chemistry Chemical Physics, vol. 10, no. 44, pp. 6615-6620, 2008.

[79] É. Brémond, A. J. Pérez-Jiménez, J. C. Sancho-García, and C. Adamo, "Range-separated hybrid density functionals made simple," The Journal of Chemical Physics, vol. 150, no. 20, 201102.

[80] A. Toro-Labbé, Ed., Theoretical Aspects of Chemical Reactivity, Elsevier Science, Amsterdam, Netherlands, 2007.

[81] C. Morell, A. Grand, and A. Toro-Labbé, "New dual descriptor for chemical reactivity," Journal of Physical Chemistry A, vol. 109, pp. 205-212, 2005.

[82] C. Morell, A. Grand, and A. Toro-Labbé, "Theoretical support for using the $\Delta \mathrm{f}(\mathrm{r})$ descriptor," Chemical Physics Letters, vol. 425, no. 4-6, pp. 342-346, 2006.

[83] J. I. Martínez-Araya, "Revisiting caffeate's capabilities as a complexation agent to silver cation in mining processes by means of the dual descriptor-a conceptual DFT approach," Journal of Molecular Modeling, vol. 18, no. 9, pp. 4299-4307, 2012.

[84] J. I. Martínez-Araya, "Explaining reaction mechanisms using the dual descriptor: a complementary tool to the molecular electrostatic potential," Journal of Molecular Modeling, vol. 19, no. 7, pp. 2715-2722, 2012.

[85] J. I. Martínez-Araya, "Why is the dual descriptor a more accurate local reactivity descriptor than Fukui functions?" Journal of Mathematical Chemistry, vol. 53, no. 2, pp. 451-465, 2015.

[86] S. Esvari Jujjavarapu, S. Dhagat, and M. Yadav, ComputerAided Design of Antimicrobial Lipopeptides as Prospective Drug Candidates, CRC Press LLC, Boca Raton, FL, USA, 2019. 